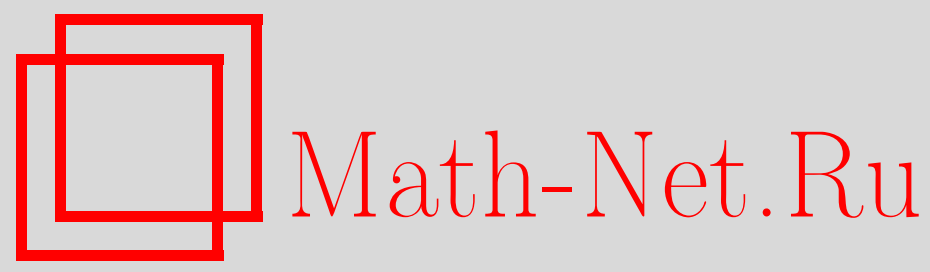

Г. Ю. Куликов, Асимптотические оценки погрешности метода простых итераций, модифицированного и обобщенного методов Ньютона, Матем. заметки, 1998, том 63, выпуск $4,562-571$

DOI: https://doi.org/10.4213/mzm1316

Использование Общероссийского математического портала Math-Net.Ru подразумевает, что вы прочитали и согласны с пользовательским соглашением http://www.mathnet.ru/rus/agreement

Параметры загрузки:

IP : 54.198 .187 .58

26 апреля 2023 г., 16:18:56

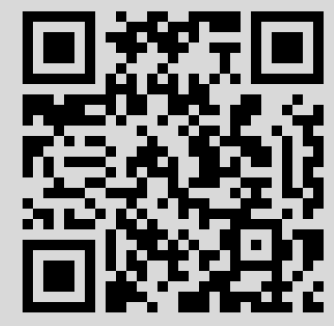




\title{
АСИМПТОТИЧЕСКИЕ ОЦЕНКИ ПОГРЕШНОСТИ МЕТОДА ПРОСТЫХ ИТЕРАЦИЙ, МОДИФИЦИРОВАННОГО И ОБОБШЕННОГО МЕТОДОВ НЬЮТОНА
}

\author{
Г. Ю. Куликов
}

\begin{abstract}
В статье изучаются асимптотические оценки погрешности метода простых итераций, модифицированного и обобщенного методов Ньютона. Такие результаты в отличие от классических дают явную оценку ошибки этих итерационных процессов через их параметры, что играет решающую роль не только при доказательстве сходимости комбинированных методов, но и для определения порядка сходимости. Кроме того, на практике это дает возможность теоретически оценить число итераций, достаточное для построения комбинированного метода максимального порядка, а значит, определить оптимальное число итераций.

Библиография: 10 названий.
\end{abstract}

1. Введение. Решение многих задач современной прикладной математики часто оказьвается возможньп только при использовании тех или иных неявных вычислительных алгоритмов, так как такие методы более устойчивы, чем явные [1]. С другой стороны, в результате применения неявных методов к нелинейньм задачам мы получаем системы нелинейных алгебраических уравнений, точное решение которых в обшем случае не может быть найдено. Таким образом, мы вьнуждены применять итерационные методы для решения систем нелинейных уравнений, которые вносят дополнительную погрешность, что в свою очередь может сказаться на точности решения поставленной задачи. Чаще всего в качестве таких методов используется метод простьх итераций, модифицированньй и полный методы Ньютона, а также другие итерационные методы ньютоновского типа [2].

Теорема Канторовича [2, с. 404] дает замечательную оценку погрешности полного метода Ньютона и позволяет в явном виде оценить ошибку через параметры этого метода. Поэтому на практике достаточно оценить эти параметры через параметры задачи, чтобы получить полную оценку погрешности приближенного решения. Применение полного метода Ньютона для решения систем нелинейных дифференциально-алгебраических уравнений можно найти в [3]-[5].

K сожалению, из-за значительных затрат компьютерного времени полньй вариант метода Ньютона используется крайне редко, а результаты сходимости метода простых итераций, модифицированного и обобщенного методов Ньютона не дают явной оценки погрешности этих методов [2, с. 401-408], что существенно затрудняет получение полной оценки погрешности приближенного решения. Так, в [6] доказательство сходимости комбинированного метода Эйлера - простых итераций - носит достаточно сложный 
характер. Более того, подход, предложенньй в [6], позволяет получить оценку только первого порядка точности относительно размера шага численного интегрирования, так как более точная оценка означает учет членов более высокого порядка при разложении ошибки в ряд Тейлора и вряд ли возможна на практике. Таким образом, отсутствие явных оценок для указанных выше итерационных методов является серьезным препятствием для построения комбинированных методов высокого порядка сходимости.

Конечно, некоторые явные оценки погрешностей итерационных методов ньютоновского типа можно найти в [7]. В частности, теорема 6 в [7, с. 680] дает явную оценку модифицированного метода Ньютона. Однако, нетрудно видеть, что эта теорема, как и другие результаты такого рода в [7], требует существования ограниченной второй производной у отображения, задающего систему нелинейных алгебраических уравнений, что значительно сужает применимость этих результатов на практике. Более того, ниже мы покажем, что неявная оценка погрешности модифищированного метода Ньютона в $[2$, с. 404$]$ точнее явной оценки в $[7$, с. 680$]$. Поэтому получение асимптотических разложений ошибки метода простых итераций, модифищированного и обобщенного методов Ньютона при наиболее слабых предположениях о гладкости системы алгебраических уравнений является актуальной задачей. Такие результаты позволяют получить явные оценки ошибки через параметры этих методов и, следовательно, являются хорошим средством доказательства сходимости комбинированных методов.

2. Метод простых итераций. Предположим, что задана система нелинейных алгебраических уравнений

$$
x=G x,
$$

где $x \in \mathbb{R}^{s}, G: D \subset \mathbb{R}^{s} \rightarrow \mathbb{R}^{s}$. Тогда метод простых итераций имеет вид

$$
x^{N}=G x^{N-1}, \quad N=1,2, \ldots,
$$

и $x^{0}$ - некоторое начальное приближение. В этом случае справедлива следующая теорема о методе простых итераций [2, с. 401].

Теорема 1. Пусть отображсение $G: D \subset \mathbb{R}^{s} \rightarrow \mathbb{R}^{s} \quad F$-дифферениируемо на выпуклом мноэсестве $D_{0} \subset D$ и производная этого отображсения непрерывна по Juпшииу, m.e.

$$
\left\|\partial G\left(x^{\prime}\right)-\partial G\left(x^{\prime \prime}\right)\right\| \leqslant \gamma\left\|x^{\prime}-x^{\prime \prime}\right\| \quad \forall x^{\prime}, x^{\prime \prime} \in D_{0} .
$$

Предположим, что существует точка $x^{0} \in D_{0}$ такая, что $\left\|\partial G\left(x^{0}\right)\right\| \leqslant \delta<1 u$ $\alpha=\gamma \eta /(1-\delta)^{2}<1 / 2$, где $\eta \geqslant\left\|x^{0}-G x^{0}\right\|$. Положим

$$
p^{*}=\frac{1-\delta}{\gamma}\left(1-(1-2 \alpha)^{1 / 2}\right), \quad p^{* *}=\frac{1-\delta}{\gamma}\left(1+(1-2 \alpha)^{1 / 2}\right)
$$

и допустим, что замкнутый шар $\bar{S}\left(x^{0}, p^{*}\right) \subset D_{0}$. Тогда итерации (2) лежсат в $\bar{S}\left(x^{0}, p^{*}\right)$ и сходятся $\kappa$ единственному решению $x^{*}$ системы $(1)$ в $S\left(x^{0}, p^{* *}\right) \cap D_{0}$. При этом справедлива оченка ошибки

$$
\left\|x^{*}-x^{N}\right\| \leqslant p^{*}-p_{N}, \quad N=1,2, \ldots,
$$

где $p_{N}=\frac{1}{2} \gamma p_{N-1}^{2}+\delta p_{N-1}+\eta, p_{0}=0$.

Учитывая неявньй вид оценки (3), докажем теорему об асимптотическом разложении ошибки метода простых итераций при $\alpha \rightarrow 0$. 
Теорема 2. Пусть выполнены условия теоремы 1. Тогда для оиибки метода простых итераций при достаточно малом а справедлива оченка

$$
\left\|x^{*}-x^{N}\right\| \leqslant \sum_{i=1}^{N} R_{i}(N)(2 \alpha)^{i}+O\left((2 \alpha)^{N+1}\right), \quad N=1,2, \ldots
$$

$2 \partial e$

$$
R_{i}(N)=\delta R_{i}(N-1)+(1-\delta) \sum_{l=1}^{i-1} \frac{\prod_{m=1}^{l-1}(m-1 / 2)}{l !} R_{i-l}(N-1)
$$

и $R_{i+1}(i), i=0,1, \ldots, N-1,-$ некоторые положительные константы.

ДокАЗАТЕЛЬСТво. Покажем, что

$$
p^{*}-p_{N} \leqslant \sum_{i=1}^{N} R_{i}(N)(2 \alpha)^{i}+O\left((2 \alpha)^{N+1}\right)
$$

Тогда (4) будет следовать из (3) и (6).

Доказательство проведем по индукции.

1) Пусть $N=1$. Тогда

$$
\begin{aligned}
p^{*}-p_{1} & =\frac{1-\delta}{\gamma}\left(1-(1-2 \alpha)^{1 / 2}\right)-\eta=\frac{1-\delta}{\gamma}\left(1-\left(1-\frac{\gamma}{(1-\delta)^{2}} \eta+O\left((2 \alpha)^{2}\right)\right)\right)-\eta \\
& =\frac{\delta}{1-\delta} \eta+O\left((2 \alpha)^{2}\right)=\frac{\delta(1-\delta)}{2 \gamma} 2 \alpha+O\left((2 \alpha)^{2}\right)
\end{aligned}
$$

Таким образом, при $N=1$ формула (6) справедлива (мы считаем сумму равной 0 , если нижний индекс суммирования больше верхнего) и $R_{1}(0)=(1-\delta) /(2 \gamma)$.

2 ) Пусть оценка (6) верна при $N=1,2, \ldots, j$. Докажем, что она верна и при $N=j+1$. Из определения последовательности $\left\{p_{N}\right\}$ следует

$$
p^{*}-p_{j+1}=p^{*}-\frac{1}{2} \gamma p_{j}^{2}-\delta p_{j}-\eta
$$

Принимая во внимание предположение индукции при $N=j$, имеем

$$
p_{j} \geqslant p^{*}-\sum_{i=1}^{j} R_{i}(j)(2 \alpha)^{i}-O\left((2 \alpha)^{j+1}\right) .
$$

Теперь покажем, что при достаточно малом $\alpha$ обе части формулы (9) неотрицательны. Для левой части этой формулы утверждение очевидно в силу построения последовательности $\left\{p_{N}\right\}$. Докажем, что оно справедливо и для правой.

Раскладьвая $p^{*}$ в ряд Тейлора в окрестности $\alpha=0$, получаем

$$
p^{*}=\frac{1-\delta}{2 \gamma}(2 \alpha)+O\left((2 \alpha)^{2}\right) \text {. }
$$


Отсюда следует, что при $\alpha \rightarrow 0$ представляют интерес только члены первого порядка в правой части формулы (9). Таким образом, нам достаточно убедиться, что

$$
\frac{1-\delta}{2 \gamma} \geqslant R_{1}(N), \quad N=1,2, \ldots, j
$$

Из (5) и (7) имеем

$$
R_{1}(N)=\frac{\delta^{N}(1-\delta)}{2 \gamma}, \quad N=1,2, \ldots, j
$$

и (11) есть простое следствие условия $\delta<1$.

Возводя в квадрат обе части формулы (9), с учетом (10) мы получаем

$$
\begin{aligned}
p_{j}^{2} \geqslant & \left(p^{*}-\sum_{i=1}^{j} R_{i}(j)(2 \alpha)^{i}-O\left((2 \alpha)^{j+1}\right)\right)^{2} \\
= & p^{* 2}+\left(\sum_{i=1}^{j} R_{i}(j)(2 \alpha)^{i}\right)^{2}+O\left((2 \alpha)^{2 j+2}\right)-2 p^{*} \sum_{i=1}^{j} R_{i}(j)(2 \alpha)^{i}-2 p^{*} O\left((2 \alpha)^{j+1}\right) \\
& +2 O\left((2 \alpha)^{j+1}\right) \sum_{i=1}^{j} R_{i}(j)(2 \alpha)^{i} \\
& =p^{* 2}+\left(\sum_{i=1}^{j} R_{i}(j)(2 \alpha)^{i}\right)^{2}-2 p^{*} \sum_{i=1}^{j} R_{i}(j)(2 \alpha)^{i}-O\left((2 \alpha)^{j+2}\right) .
\end{aligned}
$$

С другой стороны, для $p^{* 2}$ справедливо

$$
\begin{aligned}
p^{* 2} & =\left(\frac{1-\delta}{\gamma}\left(1-(1-2 \alpha)^{1 / 2}\right)\right)^{2}=\frac{(1-\delta)^{2}}{\gamma^{2}}\left(1-2(1-2 \alpha)^{1 / 2}+1-2 \alpha\right) \\
& =2 \frac{(1-\delta)^{2}}{\gamma^{2}}\left(1-(1-2 \alpha)^{1 / 2}\right)-\frac{2 \eta}{\gamma}=\frac{2(1-\delta)}{\gamma} p^{*}-\frac{2 \eta}{\gamma} .
\end{aligned}
$$

Подставляя (14) в (13), а затем (13) в (8), получаем оценку

$$
\begin{aligned}
p^{*}-p_{j+1} \leqslant & p^{*}-\frac{1}{2} \gamma\left(p^{* 2}+\left(\sum_{i=1}^{j} R_{i}(j)(2 \alpha)^{i}\right)^{2}-2 p^{*} \sum_{i=1}^{j} R_{i}(j)(2 \alpha)^{i}-O\left((2 \alpha)^{j+2}\right)\right) \\
& \quad-\delta p^{*}+\delta \sum_{i=1}^{j} R_{i}(j)(2 \alpha)^{i}+\delta O\left((2 \alpha)^{j+1}\right)-\eta \\
\leqslant & p^{*}-(1-\delta) p^{*}+\eta-\frac{1}{2} \gamma\left(\sum_{i=1}^{j} R_{i}(j)(2 \alpha)^{i}\right)^{2}+\gamma p^{*} \sum_{i=1}^{j} R_{i}(j)(2 \alpha)^{i} \\
& -\delta p^{*}+\delta \sum_{i=1}^{j} R_{i}(j)(2 \alpha)^{i}+\delta R_{j+1}(j)(2 \alpha)^{j+1}-\eta+O\left((2 \alpha)^{j+2}\right)
\end{aligned}
$$




$$
\begin{aligned}
& \leqslant \delta \sum_{i=1}^{j+1} R_{i}(j)(2 \alpha)^{i}+(1-\delta)\left(\sum_{i=1}^{j} \frac{\prod_{m=1}^{i-1}(m-1 / 2)}{i !}(2 \alpha)^{i}\right)\left(\sum_{i=1}^{j} R_{i}(j)(2 \alpha)^{i}\right) \\
& \quad+O\left((2 \alpha)^{j+2}\right) \\
& =\sum_{i=1}^{j+1} R_{i}(j+1)(2 \alpha)^{i}+O\left((2 \alpha)^{j+2}\right),
\end{aligned}
$$

где

$$
R_{i}(j+1)=\delta R_{i}(j)+(1-\delta) \sum_{l=1}^{i-1} \frac{\prod_{m=1}^{l-1}(m-1 / 2)}{l !} R_{i-l}(j)
$$

откуда следует (6) и теорема 2.

Теорема 2 дает хорошую асимптотическую оценку ошибки метода простых итераций, единственным недостатком является лишь сложный вид коэффициентов $R_{i}(N)$. Поэтому далее покажем, что эти коэффициенты стремятся к 0 со скоростью геометрической прогрессии.

ТЕОрема 3. Пусть выполнены условия теоремы 1. Тогда для ошибки метода

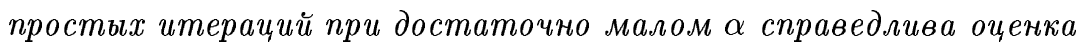

$$
\left\|x^{*}-x^{N}\right\| \leqslant C \sum_{i=1}^{N} \delta^{N-i+1}(2 \alpha)^{i}, \quad N=1,2, \ldots,
$$

әде $C$ - некоторая константа.

ДокАЗАТЕЛЬСтво. Применяя теорему 2, мы получаем оценку (4). Таким образом, нам осталось показать, что

$$
R_{i}(N)=O\left(\delta^{N-i+1}\right), \quad N=1,2, \ldots, \quad i=1,2, \ldots, N
$$

Для доказательства используем индукцию по $i$.

1) Пусть $i=1$. Тогда (15) следует из (12).

2) Пусть формула (15) верна при $i=1,2, \ldots, j$. Докажем, что она верна и при $i=j+1$.

В силу формулы (5), предположения индукции и очевидного неравенства

$$
\frac{\prod_{m=1}^{l-1}(m-1 / 2)}{l !} \leqslant 1, \quad l=1,2, \ldots
$$

мы получаем для любого $N \geqslant j+1$

$$
\begin{aligned}
R_{j+1}(N) & =\delta R_{j+1}(N-1)+(1-\delta) \sum_{l=1}^{j} O\left(\delta^{N-l}\right)=\delta R_{j+1}(N-1)+O\left(\delta^{N-j}\right) \\
& =\delta\left(\delta R_{j+1}(N-2)+O\left(\delta^{N-j-1}\right)\right)+O\left(\delta^{N-j}\right) \\
& =\delta^{2} R_{j+1}(N-2)+O\left(\delta^{N-j}\right)=\cdots=\delta^{N-j} R_{j+1}(j)+O\left(\delta^{N-j}\right)=O\left(\delta^{N-j}\right),
\end{aligned}
$$

так как $R_{j+1}(j)$ - некоторая положительная константа. Теорема доказана. 
3. Модифицированный метод Ньютона. Чтобы применить модифицированный метод Ньютона для решения системы (1), надо предварительно привести ее к следующему виду:

$$
F x=0,
$$

где $F=E-G$, а $E$ - единичная матрица размера $s$. Тогда итерационньй процесс

$$
x^{N}=x^{N-1}-\partial F\left(x^{0}\right)^{-1} F x^{N-1}, \quad N=1,2, \ldots,
$$

назьвается модифицированным методом Ньютона. Для этого метода справедлива следующая теорема [2, с. 404].

Теорема 4. Пусть отображсение $F: D \subset \mathbb{R}^{s} \rightarrow \mathbb{R}^{s} \quad F$-дифференцируемо на выпуклом мнохсестве $D_{0} \subset D$ и производная этого отображсения непрерывна по Липшичу с постоянной $\gamma$. Предполохим, что существует точка $x^{0} \in D_{0}$ такая, что $\left\|\partial F\left(x^{0}\right)^{-1}\right\| \leqslant \beta$ и $\alpha=\beta \gamma \eta \leqslant 1 / 2$, где $\eta \geqslant\left\|\partial F\left(x^{0}\right)^{-1} F x^{0}\right\|$. Положим

$$
p^{*}=(\beta \gamma)^{-1}\left(1-(1-2 \alpha)^{1 / 2}\right), \quad p^{* *}=(\beta \gamma)^{-1}\left(1+(1-2 \alpha)^{1 / 2}\right)
$$

и допустим, что замкнутый шар $\bar{S}\left(x^{0}, p^{*}\right) \subset D_{0}$. Тогда итерачии (17) определены корректно, лежат в $\bar{S}\left(x^{0}, p^{*}\right)$ и сходятся $\kappa$ единственному решению $x^{*}$ системы (16) в $S\left(x^{0}, p^{* *}\right) \cap D_{0}$. При этом имеет место оченка оиибки

$$
\left\|x^{*}-x^{N}\right\| \leqslant p^{*}-p_{N}, \quad N=1,2, \ldots,
$$

əде $p_{N}=\frac{1}{2} \beta \gamma p_{N-1}^{2}+\eta, p_{0}=0$.

Как мы отмечали выше, неявньй вид оценки (18) существенно затрудняет ее использование на практике. Поэтому если дополнительно потребовать, чтобы отображение $F$ имело ограниченную вторую производную, то теорема 6 из $[7$, с. 680$]$ приводит к явной оценке ошибки модифицированного метода Ньютона

$$
\left\|x^{*}-x^{N}\right\| \leqslant(\beta \gamma)^{-1}\left(1-(1-2 \alpha)^{1 / 2}\right)^{N+1}, \quad N=1,2, \ldots,
$$

где $\left\|\partial^{2} F(x)\right\| \leqslant \gamma$ на множестве $D_{0}$, а $\alpha, \beta$ и $\eta$ имеют тот же смысл, что и в теореме 4.

Хотя оценка (19) и имеет явньй вид, она доказана только при более жестких условиях, налагаемых на систему (16), чем оценка (18). Более того, мы покажем, что оценка (18) точнее оценки (19), т.е.

$$
p^{*}-p_{N}<(\beta \gamma)^{-1}\left(1-(1-2 \alpha)^{1 / 2}\right)^{N+1}, \quad N=1,2, \ldots
$$

Воспользуемся индукцией.

1) Пусть $N=1$. Тогда

$$
\begin{aligned}
p^{*}-p_{1} & =(\beta \gamma)^{-1}\left(1-(1-2 \alpha)^{1 / 2}\right)-\eta<2\left((\beta \gamma)^{-1}\left(1-(1-2 \alpha)^{1 / 2}\right)-\eta\right) \\
& =(\beta \gamma)^{-1}\left(1-(1-2 \alpha)^{1 / 2}\right)^{2} .
\end{aligned}
$$

Откуда следует, что (20) при $N=1$ вьполнено.

2) Предположим теперь, что (20) справедливо при $N=1,2, \ldots, j$, и докажем, что эта формула имеет место и при $N=j+1$. 
Используя определение последовательности $\left\{p_{N}\right\}$ из (18), предположение индукции и формулу для $p^{* 2}$ (см. [8, формула (4.7)]), мы получаем (20) при $N=j+1$

$$
\begin{aligned}
p^{*}-p_{j+1} & =p^{*}-\frac{1}{2} \beta \gamma p_{j}^{2}-\eta<p^{*}-\frac{1}{2} \beta \gamma\left(p^{*}-(\beta \gamma)^{-1}\left(1-(1-2 \alpha)^{1 / 2}\right)^{j+1}\right)^{2}-\eta \\
& =p^{*}-\eta-\frac{1}{2} \beta \gamma p^{* 2}+p^{*}\left(1-(1-2 \alpha)^{1 / 2}\right)^{j+1}-(\beta \gamma)^{-2}\left(1-(1-2 \alpha)^{1 / 2}\right)^{2 j+2} \\
& <(\beta \gamma)^{-1}\left(1-(1-2 \alpha)^{1 / 2}\right)^{j+2} .
\end{aligned}
$$

В результате мы не только доказали, что оценка (18) точнее оценки (19), но и выяснили, что последняя остается справедливой и при более слабых предположениях относительно гладкости задачи (16).

ТЕОРема 5. Пусть выполнены условия теоремы 4. Тогда для ошибки модифииированного метода Ньютона справедлива оценка (19).

Следствием теоремы 5 является тот факт, что при $\alpha \rightarrow 0$ погрешность на $N$-й итерации модифицированного метода Ньютона ведет себя, по крайней мере, как $O\left(\alpha^{N+1}\right)$. Однако, с учетом (20) интересно выяснить асимптотическое поведение погрешности модифицированного метода Ньютона для более точной оценки (18).

ТЕОрема 6. Пусть выполнены условия теоремы 4. Тогда для ошибки модифииированного метода Ньютона при достаточно малом а справедлива оченка

$$
\left\|x^{*}-x^{N}\right\| \leqslant \mathrm{C} \alpha^{N+1}, \quad N=1,2, \ldots,
$$

әде $C$-некоторая константа.

ДокАЗАТЕЛьСтво. Оценка (21) сразу следует из (18) и представления

$$
p^{*}-p_{N}=O\left(\alpha^{N+1}\right), \quad N=1,2, \ldots,
$$

при $\alpha \rightarrow 0$. Справедливость (22) нетрудно проверить по индукции [8]. Теорема доказана.

Итак, мы показали, что хотя оценка погрешности модифицированного метода Ньютона (18) точнее (19), обе оценки являются асимптотически эквивалентными, так как при достаточно малом $\alpha$ дают сходимость модифицированного метода Ньютона со скоростью геометрической прогрессии.

В заключение этого пункта отметим, что оценка (21) хорошо соответствует оценке ошибки полного метода Ньютона. Из теоремы Канторовича следует, что при одной итерации ошибка полного метода Ньютона ведет себя как $O\left(\alpha^{2}\right)$ при $\alpha \rightarrow 0$. С другой стороны, при одной итерации полньй и модифицированньй методы Ньютона совпадают, поэтому они должны иметь одинаковую погрешность, что следует из (21) при $N=1$.

4. Обобщенный метод Ньютона. Обобщенным методом Ньютона назьвается итерационньй процесс следующего вида:

$$
x^{N}=x^{N-1}-A\left(x^{N-1}\right)^{-1} F x^{N-1}, \quad N=1,2, \ldots,
$$

где $A$ - некоторая квадратная матрица размера $s$. Для методов такого типа справедлива следующая теорема [2, с. 408]. 
Теорема 7. Пусть отображсение $F: D \subset \mathbb{R}^{s} \rightarrow \mathbb{R}^{s} \quad F$-дифферениируемо на выпуклом множестве $D_{0} \subset D$ и производная этого отображения непрерывна по Липшицу с постоянной $\gamma$. Пусть $A: D_{0} \subset \mathbb{R}^{s} \rightarrow L\left(\mathbb{R}^{s}\right)$, где $L\left(\mathbb{R}^{s}\right)$ - пространство линейных операторов в $\mathbb{R}^{s}, u x^{0} \in D_{0}$ таковы, что при $\delta_{0}, \delta_{1} \geqslant 0$

$$
\left\|A(x)-A\left(x^{0}\right)\right\| \leqslant \mu\left\|x-x^{0}\right\|, \quad\|\partial F(x)-A(x)\| \leqslant \delta_{0}+\delta_{1}\left\|x-x^{0}\right\| \quad \forall x \in D_{0} .
$$

Предположим также, что матрица $A\left(x^{0}\right)$ невырождена и

$$
\left\|A\left(x^{0}\right)^{-1}\right\| \leqslant \beta, \quad\left\|A\left(x^{0}\right)^{-1} F x^{0}\right\| \leqslant \eta,
$$

причем $\beta \delta_{0}<1$ и $\alpha=\sigma \beta \gamma \eta /\left(1-\beta \delta_{0}\right)^{2} \leqslant 1 / 2$, әде $\sigma=\max \left\{1,\left(\mu+\delta_{1}\right) / \gamma\right\}$. Положим

$$
p^{*}=\frac{1-\beta \delta_{0}}{\sigma \beta \gamma}\left(1-(1-2 \alpha)^{1 / 2}\right), \quad p^{* *}=\frac{1-\beta \delta_{0}}{\sigma \beta \gamma}\left(1+(1-2 \alpha)^{1 / 2}\right)
$$

и допустим, что замкнутый шар $\bar{S}\left(x^{0}, p^{*}\right) \subset D_{0}$. Тогда итерации (23) определены корректно, лежат в $\bar{S}\left(x^{0}, p^{*}\right)$ и сходятся $\kappa$ единственному решению $x^{*}$ системы (16) в $S\left(x^{0}, p^{* *}\right) \cap D_{0}$. При этом справедлива оченка

$$
\left\|x^{*}-x^{N}\right\| \leqslant p^{*}-p_{N}, \quad N=1,2, \ldots,
$$

əде последовательность $\left\{p_{N}\right\}$ определяется следующим образом:

$$
\begin{gathered}
p_{N+1}-p_{N}=\frac{1}{1-\beta \mu p_{N}}\left(\frac{1}{2} \sigma \beta \gamma\left(p_{N}-p_{N-1}\right)+\beta \delta_{0}+\beta(\sigma \gamma-\mu) p_{N-1}\right)\left(p_{N}-p_{N-1}\right), \\
N=1,2, \ldots, \quad p_{0}=0, \quad p_{1}=\eta .
\end{gathered}
$$

Учитывая неявный вид оценки (24), докажем теорему об асимптотическом разложении погрешности обобшенного метода Ньютона.

ТЕоРема 8. Пусть выполнены условия теоремы 7. Тогда для ошибки обобщенного метода Ньютона при достаточно малом $\eta$ справедлива оченка

$$
\left\|x^{*}-x^{N}\right\| \leqslant \frac{\left(\beta \delta_{0}\right)^{N}}{1-\beta \delta_{0}} \eta+O\left(\eta^{2}\right), \quad N=1,2, \ldots
$$

ДокАЗАТЕЛЬСТво. Нам достаточно показать, что

$$
p^{*}-p_{N}=\frac{\left(\beta \delta_{0}\right)^{N}}{1-\beta \delta_{0}} \eta+O\left(\eta^{2}\right), \quad N=1,2, \ldots,
$$

при $\eta \rightarrow 0$.

Доказательство проведем по индукции.

1) Пусть $N=1$. Тогда из (25) имеем

$$
\begin{aligned}
p^{*}-p_{1} & =\frac{1-\beta \delta_{0}}{\sigma \beta \gamma}\left(1-(1-2 \alpha)^{1 / 2}\right)-\eta \\
& =\frac{1-\beta \delta_{0}}{\sigma \beta \gamma}\left(1-\left(1-\frac{\sigma \beta \gamma}{\left(1-\beta \delta_{0}\right)^{2}} \eta+O\left(\eta^{2}\right)\right)\right)-\eta \\
& =\frac{1}{1-\beta \delta_{0}} \eta-\eta+O\left(\eta^{2}\right)=\frac{\beta \delta_{0}}{1-\beta \delta_{0}} \eta+O\left(\eta^{2}\right) .
\end{aligned}
$$


Таким образом, при $N=1$ формула (27) верна.

2) Предположим, что асимптотическое разложение (27) верно при $N=1,2, \ldots, j$. Докажем, что эта формула верна и при $N=j+1$.

Прежде всего покажем, что справедливо асимптотическое разложение

$$
p_{N}-p_{N-1}=\left(\beta \delta_{0}\right)^{N-1} \eta+O\left(\eta^{2}\right), \quad N=1,2, \ldots
$$

Снова воспользуемся индукцией.

1) Пусть $N=1$. Тогда $p_{1}-p_{0}=\eta$ и формула (28) верна.

2) Пусть (28) справедливо при $N=1,2, \ldots, k$. Докажем, что это разложение имеет место и при $N=k+1$, где $k+1 \leqslant j$.

Принимая во внимание предположение индукции и (25), мы получаем

$$
\begin{aligned}
& p_{k+1}-p_{k} \\
& =\frac{1}{1-\beta \mu p_{k}}\left(\frac{1}{2} \sigma \beta \gamma\left(\left(\beta \delta_{0}\right)^{k-1} \eta+O\left(\eta^{2}\right)\right)+\beta \delta_{0}+\beta(\sigma \gamma-\mu) p_{k-1}\right)\left(\left(\beta \delta_{0}\right)^{k-1} \eta+O\left(\eta^{2}\right)\right) \\
& =\left(\frac{1}{2} \sigma \beta \gamma\left(\beta \delta_{0}\right)^{k-1} \eta+\beta \delta_{0}+\beta(\sigma \gamma-\mu) p_{k-1}\right)\left(1+\sum_{i=1}^{\infty}\left(\beta \mu p_{k}\right)^{i}\right)\left(\beta \delta_{0}\right)^{k-1} \eta+O\left(\eta^{2}\right)
\end{aligned}
$$

При $i=1,2, \ldots, j$ из (27) следует

$$
p_{i}=p^{*}-\frac{\left(\beta \delta_{0}\right)^{i}}{1-\beta \delta_{0}} \eta+O\left(\eta^{2}\right), \quad N=1,2, \ldots
$$

Раскладывая $p^{*}$ в ряд Тейлора в окрестности точки $\eta=0$, из (30) получаем

$$
p_{i}=\frac{1-\left(1-\beta \delta_{0}\right)\left(\beta \delta_{0}\right)^{i}}{1-\beta \delta_{0}} \eta+O\left(\eta^{2}\right), \quad i=1,2, \ldots, j .
$$

Тогда, подставляя (31) в (29), мы приходим к (28) при $N=k+1$. Таким образом, разложение $(28)$ доказано.

Теперь докажем справедливость (27) при $N=j+1$. Принимая во внимание предположение индукции, (25), (28) и (31), имеем

$$
\begin{aligned}
p^{*}-p_{j+1}= & p^{*}-p_{j}-\frac{1}{1-\beta \mu p_{j}}\left(\frac{1}{2} \sigma \beta \gamma\left(p_{j}-p_{j-1}\right)+\beta \delta_{0}+\beta(\sigma \gamma-\mu) p_{j-1}\right)\left(p_{j}-p_{j-1}\right) \\
= & \frac{\left(\beta \delta_{0}\right)^{j}}{1-\beta \delta_{0}} \eta+O\left(\eta^{2}\right)-\left(\frac{1}{2} \sigma \beta \gamma\left(\left(\beta \delta_{0}\right)^{j-1} \eta+O\left(\eta^{2}\right)\right)+\beta \delta_{0}\right. \\
& \left.+\beta(\sigma \gamma-\mu)\left(\frac{1-\left(1-\beta \delta_{0}\right)\left(\beta \delta_{0}\right)^{j-1}}{1-\beta \delta_{0}} \eta+O\left(\eta^{2}\right)\right)\right) \\
& \quad \times\left(\left(\beta \delta_{0}\right)^{j-1} \eta+O\left(\eta^{2}\right)\right) \sum_{i=0}^{\infty}\left(\frac{1-\left(1-\beta \delta_{0}\right)\left(\beta \delta_{0}\right)^{j}}{1-\beta \delta_{0}} \eta+O\left(\eta^{2}\right)\right)^{i} \\
= & \frac{\left(\beta \delta_{0}\right)^{j}}{1-\beta \delta_{0}} \eta-\left(\beta \delta_{0}\right)^{j} \eta+O\left(\eta^{2}\right)=\frac{\left(\beta \delta_{0}\right)^{j+1}}{1-\beta \delta_{0}} \eta+O\left(\eta^{2}\right) .
\end{aligned}
$$

Формула (27) доказана. Тогда (26) непосредственно следует из (24) и (27). Теорема доказана. 
5. Заключение. Итак, в этой статье мы получили асимптотические оценки погрешности метода простых итераций, модифицированного и обобщенного методов Ньютона. Такие результаты в отличие от классических дают явную оценку ошибки этих итерационных процессов, что играет решающую роль не только при доказательстве сходимости комбинированных методов, но и для определения порядка сходимости. Кроме того, на практике это дает возможность теоретически оценить число итераций, достаточное для построения комбинированного метода максимального порядка, а значит, определить оптимальное число итераций. Последнее является, в свою очередь, необходимым для построения эффективньх вычислительных программ. Такие результаты, касающиеся численных методов решения систем дифференциально-алгебраических уравнений можно найти в [9]. Более того, асимптотические оценки погрешности указанных итерационных методов являются необходимыми для обоснования неявных численных методов с автоматическим выбором шага интегрирования [10].

Следует отметить, что асимптотическая оценка погрешности обобщенного метода Ньютона имеет особое значение. Во-первых, использование в итерационном процесce (23) матрицы $A$, отличной от точного якобиана системы (16), позволяет существенно облегчить обращение этой матрицы и, как следствие, сократить время решения задачи. Во-вторых, это позволяет находить решение задачи (16) даже в случае, если якобиан такой системы является плохо обусловленной матрищей, что чрезвычайно важно для решения систем дифференциально-алгебраических уравнений высокого индекса.

\section{СПИСОК ЦИТИРОВАННОЙ ЛИТЕРАТУРЫ}

[1] Hairer E., Wanner G. Solving Ordinary Differential Equations. II: Stiff and Differential-Algebraic Problems. Springer Ser. Comput. Math. V. 14. Berlin: Springer, 1991.

[2] Ортега Дж., Рейнболдт В. Итерационные методы решения нелинейных систем уравнений со многими неизвестными. М.: Мир, 1975.

[3] Куликов Г. Ю. Об одном способе численного решения автономной задачи Коши с алгебраической связью на фазовые переменные // Вестн. МГУ. Сер. 1. Матем., мех. 1992. № 1. C. $14-19$.

[4] Куликов Г. Ю. О численном решении автономной задачи Коши с алгебраической связью на фазовые переменные (невырожденный случай) // Вестн. МГУ. Сер. 1. Матем., мех. 1993. № 3. C. $10-14$.

[5] Куликов Г. Ю. Численное решение задачи Коши с алгебраической связью на фазовые переменные (с приложениями в медицинской кибернетике). Дисс. . . . к.ф.-м.н. М.: ВЦ РАН, 1994.

[6] Куликов Г. Ю. О численном решении автономной задачи Коши с алгебраической связью на фазовые переменные // ЖВМиМФ. 1993. Т. 33. № 4. С. 522-540.

[7] Канторович Л. В., Акилов Г. П. Функциональный анализ. М.: Наука, 1977.

[8] Куликов Г. Ю. Практическая реализация и эффективность численных методов решения задачи Коши с алгебраической связью // ЖВМиМФ. 1994. Т. 34. № 11. С. 1617-1631.

[9] Куликов Г. Ю. Теоремы сходимости для итеративных методов Рунге-Кутта с постоянным шагом интегрирования // ЖВМиМФ. 1996. Т. 36. № 8. С. 73-89.

[10] Kulikov G. Yu., Thomsen P. G. Convergence and implementation of implicit RungeKutta methods for DAEs. Technical Report № 7/1996. Lyngby: IMM, Technical Univ. of Denmark, 1996.

Ульяновский государственный университет

Поступило

E-mail : kulgyu@mci.univ.simbirsk.su

11.11 .96

Исправленный вариант

28.10.97 